\title{
The establishment of a practical method for the determination of piperazine residues using accelerated solvent extraction and UHPLC-FLD
}

\author{
Y.W. Guo ${ }^{1,2}$, X. Xie ${ }^{3}$, B. Wang ${ }^{2,4}$, Y.Y. Zhang ${ }^{1,2}$, K.Z. Xie ${ }^{1,2^{*}}$, X.N. Bu ${ }^{1,2}$, C.J. Liu ${ }^{1,2}$, T. Zhang ${ }^{1,2}$, \\ G.X. Zhang ${ }^{1,2}$, X.Z. Liu ${ }^{2,4}$ and G.J. Dai ${ }^{1,2}$ \\ ${ }^{1}$ College of Animal Science and Technology, Yangzhou University, Yangzhou 225009, China; ${ }^{2}$ Joint International Research \\ Laboratory of Agriculture E Agri-Product Safety, Yangzhou University, Yangzhou 225009, China; ${ }^{3}$ Institute of Veterinary Med- \\ icine, Jiangsu Academy of Agricultural Sciences, Key Laboratory of Veterinary Biological Engineering and Technology, Ministry \\ of Agriculture, Nanjing 210014, China; ${ }^{4}$ College of Veterinary Medicine, Yangzhou University, Yangzhou 225009, China
}

Corresponding Author: K.Z. Xie, yzxkz168@163.com

Received: 16 September 2019 / Accepted: 4 December 2019 / Published: 2 January 2020

(C) 2020 Codon Publications

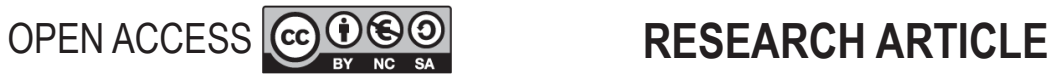

\begin{abstract}
This article describes a reliable method for estimating piperazine residues in chicken tissues (muscle, kidney and liver) and pork via ultrahigh-performance liquid chromatography (UHPLC) coupled with a fluorescence detector (FLD) using dansyl chloride (DNS-Cl) as the derivatisation reagent. After extraction by accelerated solvent extraction (ASE), the analyte was purified on a strong cation-exchange solid-phase extraction (SPE) column. Separation was achieved using an Acquity UPLC HSS T3 $(2.1 \mathrm{~mm} \times 100 \mathrm{~mm}, 1.8 \mu \mathrm{m})$ column with ultrapure water-acetonitrile $(15: 85, \mathrm{~V} / \mathrm{V})$ as the mobile phase. The results showed that when the concentration of piperazine was between the limit of quantitation (LOQ) and $200.0 \mu \mathrm{g} / \mathrm{kg}$, the peak area of the derivative had a good linear relationship with the piperazine concentration, and the coefficients of determination $\left(R^{2}\right)$ were greater than or equal to 0.9991 . When the spiked concentration of piperazine was equal to the LOQ, maximum residue limit (MRL) of 0.5, 1.0 and 2.0, the recoveries ranged from 79.64 to $99.77 \%$ and the relative standard deviations (RSDs) were $1.14-5.63 \%$. The limits of detection (LODs) and LOQs were $0.50-1.20$ and $1.80-3.50 \mu \mathrm{g} / \mathrm{kg}$, respectively. The method was applied to the quantification of piperazine residues in commercial chicken tissues and pork from local supermarkets.
\end{abstract}

Keywords: piperazine, ASE, derivatisation, UHPLC-FLD

\section{Introduction}

Coccidiosis is a parasitic disease caused by various coccidia in the genus Eimeria (Peek and Landman, 2011). Drug resistance and high outbreak and mortality rates are the main coccidiosis-related factors threatening the livestock breeding industry. Piperazine and its $\mathrm{N}$-substituted derivatives have diverse biological activities, such as antimicrobial (Marchetti et al., 2012), antihypertensive (Glamkowski et al., 1974), anticancer (Demirci et al., 2019), anti-inflammatory (Silva et al., 2015) and analgesic (Silva et al., 2015) activities, and are widely used in the treatment of coccidiosis. However, the improper use of piperazine leads to drug residues in animal-derived foods, and the long-term consumption of these contaminated products poses a serious threat to human health (Anna et al., 2017; Lee et al., 1997; Staack and Maurer, 2003). Empirical studies have found that piperazine acts as a low-potency agonist of the extrasynaptic $\gamma$-aminobutyric acid receptors in the bag region of muscle tissue (Brennan et al., 2007), that benzylpiperazine induces a reaction sensitive to dopamine antagonists (Wada et al., 2015) and that piperazine has possible carcinogenic and brain electrophysiological effects (Lin et al., 2010). Because of these potential toxic effects, the use of piperazine has been severely restricted or even prohibited 
in many countries. The European Medicines Agency (2010) and the Chinese government (Ministry of Agriculture of the People's Republic of China, 2002) set maximum residue limits (MRLs) of $400 \mu \mathrm{g} / \mathrm{kg}$ for piperazine in chicken and pork. The Food and Drug Administration (FDA) (U.S. Food and Drug Administration, 2014) stipulates an MRL of $100 \mu \mathrm{g} / \mathrm{kg}$ for piperazine in chicken and pork. The MRLs of piperazine in Japan (The Japan Food Chemical Research Foundation, 2015) are $100 \mu \mathrm{g} / \mathrm{kg}$ for chicken and $300 \mu \mathrm{g} / \mathrm{kg}$ for pork.

Various well-developed methods, including non-aqueous titration (Ciaccio et al., 1957), gravimetry (Nnaji et al., 2017), colorimetry (Lalka et al., 2010), spectrophotometry (Hayat et al., 2017), liquid chromatography (Park et al., 2016), liquid chromatography-tandem mass spectrometry (Lin et al., 2010; Xie et al., 2016) and gas chromatography-tandem mass spectrometry (Wang et al., 2017), have been reported for the measurement of piperazine and its salt residues. Nevertheless, traditional methods face many operational challenges; the processes are often quite cumbersome and time-consuming, or the detection is susceptible to substantial interference from impurities. Non-aqueous titration can be influenced by temperature, moisture and titration rate (Ciaccio et al., 1957). Gravimetry is cumbersome and has a large measurement error for low-abundance components (Nnaji et al., 2017). The applicable range of colorimetry is limited (Lalka et al., 2010). Spectrophotometry is affected by the light source, $\mathrm{pH}$, cuvette material and other factors. Chromatography is increasingly being applied in the analysis of multicomponent mixtures and complex systems. High-performance liquid chromatography-fluorescence detection (HPLCFLD) (Park et al., 2016) has been utilised to measure piperazine residues in animal-derived foods. Highperformance liquid chromatography-electrospray ionisation-tandem mass spectrometry (HPLC-ESI/MS/MS) (Lin et al., 2010) has been applied to the quantification of piperazine phosphate in human plasma. In previous studies, we developed an ultrahigh-performance liquid chromatography-electrospray ionisation-tandem triple quadrupole mass spectrometry (UHPLC-ESI/MS/MS) approach (Xie et al., 2016) and a gas chromatographyelectron ionisation-tandem mass spectrometry (GCEI/MS/MS) method (Wang et al., 2017) to determine piperazine residues.

As a result of its high sensitivity and selectivity, UHPLC is increasingly being used for quantitative studies involving biological matrices, and the use of a detector, such as an FLD, is required. The principle behind an FLD requires the solute to emit fluorescence upon exposure to ultraviolet light. Piperazine has no fluorescent group; therefore, it must be reacted with a derivatisation reagent to form a fluorescent substance. Precolumn derivatisation conditions are easily controlled, and there are many types of derivatisation reagents to choose from. In this experiment, dansyl chloride (DNS-Cl) was selected as the derivatisation reagent. The analytical method used in this study [accelerated solvent extraction (ASE) extraction and UHPLC separation] has not been reported in the literature. Based on the MRL of piperazine set by the FDA, this study established a rapid, sensitive and selective ultrahigh-performance liquid chromatography-fluorescence detector (UHPLC-FLD) method with precolumn derivatisation for the detection of piperazine residues in chicken tissues and pork. This method is of great significance for promoting the testing of the safety of animal-derived foods, protecting human health and assuring the quality of exports.

\section{Materials and methods}

\section{Chemicals and reagents}

A piperazine standard (CAS No. 110-85-0, 99.0\% purity) was purchased from Sigma-Aldrich Company (St. Louis, MO, USA). DNS-Cl (CAS No. 605-65-2, 99.0\% purity) was obtained from Yuanye Biological Technology Co., Ltd. (Thermo Fisher Scientific Inc., MA, USA). Triethylamine (99.0\% purity, chromatography grade) and acetonitrile (chromatography grade) were acquired from Thermo Fisher Scientific Inc. (MA, USA). Formic acid, n-hexane and ammonia were analytical grade and were provided by Sinopharm Chemical Reagent Corp. (Shanghai, China). The ultrapure water $\left(18.2 \mathrm{M} \Omega^{*} \mathrm{~cm}\right.$, $25^{\circ} \mathrm{C}$ ) used in this study met the standard for 'water for analytical laboratory use-specification and test methods' (Standardization Administration of the People's Republic of China, 2008).

\section{Preparation of the standard stock and working solutions}

A 10-mg portion of the piperazine standard (99.0\% purity) was accurately weighed, dissolved in acetonitrile and then transferred to a $10-\mathrm{ml}$ brown volumetric flask. After the piperazine was completely dissolved, the solution volume was adjusted to $10 \mathrm{ml}$, and it was shaken to mix evenly. Once prepared, the $1.0 \mathrm{mg} / \mathrm{ml}$ piperazine standard stock solution was sealed and stored in an ultralow-temperature refrigerator $\left(-70^{\circ} \mathrm{C}\right)$, and under these conditions, it is stable for 4 months of storage.

Piperazine standard working solutions at five concentrations $(100.0 \mu \mathrm{g} / \mathrm{ml}, 10.0 \mu \mathrm{g} / \mathrm{ml}, 1.0 \mu \mathrm{g} / \mathrm{ml}, 100.0 \mathrm{ng} /$ $\mathrm{ml}$ and $10.0 \mathrm{ng} / \mathrm{ml}$ ) were prepared by the stepwise dilution of piperazine standard stock solution $(1.0 \mathrm{mg} / \mathrm{ml})$ with acetonitrile, and the solutions were stored at $4{ }^{\circ} \mathrm{C}$ for later use. The working solutions were freshly prepared prior to every use and were used to create the standard curve. 


\section{DNS-CI solution}

The derivatisation solution $(1.0 \mathrm{mg} / \mathrm{ml})$ was prepared by dissolving $10 \mathrm{mg}$ of DNS-Cl in acetonitrile in a brown volumetric flask. The solution was adjusted to $10 \mathrm{ml}$ and then shaken thoroughly to mix, affording the DNS-Cl solution. The solution was stored in the dark and prepared fresh prior to each use.

\section{UHPLC-FLD instruments and conditions}

The separation and detection were performed on a Waters Acquity UPLC ${ }^{\mathrm{TM}}$ apparatus (Waters Corp., Milford, MA, USA) coupled with a Waters Acquity FLD ${ }^{\mathrm{TM}}$ apparatus (Waters Corp., Milford, MA, USA). The samples were separated on an Acquity UPLC HSS T3 column $(100 \mathrm{~mm} \times 2.1$ $\mathrm{mm}, 1.8 \mu \mathrm{m}$ ), and the column temperature was set at $25^{\circ} \mathrm{C}$. Mobile phase A consisted of ultrapure water, while mobile phase $B$ consisted of acetonitrile, and they were used at a ratio of $A: B=15: 85(V / V)$. The mobile phase was pumped through the column at a flow rate of $0.2 \mathrm{ml} / \mathrm{min}$. The mobile phase was passed through a $0.2-\mathrm{mm}$ nylon membrane filter before use. Experimental results indicated that the maximum excitation wavelength (Ex) and emission wavelength (Em) for the derivative were 330 and $531 \mathrm{~nm}$, respectively.

\section{Preparation of samples}

This study was conducted strictly in accordance with the requirements of the applicable ethics ordinances and was authorised by our school's ethics committee. Chicken tissues (muscle, kidney and liver) and pork samples were collected from Jinghai yellow chickens (Jinghai Poultry Company, Jiangsu, China) and Duroc-Landrace-Yorkshire swine (Kangle Farming Company, Jiangsu, China), respectively. The diet of the animals prior to slaughter was completely free of drugs. Each sample $(2.0 \pm 0.02 \mathrm{~g})$ was weighed precisely and loaded into a mortar. Diatomaceous earth $(4.0 \mathrm{~g})$ was added for homogenisation, and the mixed samples were ground as finely as possible to maximise extraction efficiency. The samples were packed in sample cells $(22 \mathrm{ml})$, and ASE was then undertaken using an ASE350 apparatus (Thermo Fisher Scientific Inc., MA, USA). The ASE procedure involved degreasing, rinsing and static extraction. The appropriate volume of $n$-hexane was pumped into the extraction cell and adequately mixed with the matrix to remove fat impurities. The sample was rinsed to remove residual solvents in the pipeline, and then the system was purged with nitrogen for $60 \mathrm{sec}$. Then two cycles of static extraction $\left(80^{\circ} \mathrm{C}, 1,500 \mathrm{psi}, 5 \mathrm{~min}\right)$ using $2 \%$ formic acid in acetonitrile as the extractant were carried to fully extract the target components.

The extracts were collected in $60-\mathrm{ml}$ collection bottles, transferred to $50-\mathrm{ml}$ centrifuge tubes and purified on a Strata-X-C SPE column (3 ml/60 mg, Phenomenex Corp., WA, USA). This SPE column not only ensures effective purification but also guarantees the appropriate flow rate. The column was activated with methanol ( $3 \mathrm{ml}$ ) and then equilibrated with $2 \%$ formic acid in water $(3 \mathrm{ml})$. After all the extracts $(20 \mathrm{ml})$ were loaded onto the column, $0.1 \mathrm{~mol} / \mathrm{L}$ aqueous hydrochloric acid $(2 \mathrm{ml})$ was loaded onto the column, and the system was then rinsed with methanol $(2 \mathrm{ml})$. Finally, the column was eluted with $10 \%$ ammonia in methanol $(9 \mathrm{ml})$, and the eluate was collected in a centrifuge tube. The eluate was concentrated under a stream of nitrogen in a nitrogen blower (No. N-EVAP-112, Organomation Corp., MD, USA). Then, the dried residue was redissolved in acetonitrile $(1 \mathrm{ml})$. The mixture was ultrasonicated with an ultrasonic wave cleaner (No. P300H, Elma Corp., Wetzikon, Switzerland) for $10 \mathrm{~min}$. Then $0.12 \%$ triethylamine $(100 \mu \mathrm{l})$ and $1.0 \mathrm{mg} / \mathrm{ml} \mathrm{DNS}-\mathrm{Cl}(600 \mu \mathrm{l})$ were sequentially added. The volume was adjusted to $2 \mathrm{ml}$ with acetonitrile, and the mixture was placed in an oven $\left(50^{\circ} \mathrm{C}, 20 \mathrm{~min}\right)$ for derivatisation. After the reaction was complete, the mixture was vortexed for $1 \mathrm{~min}$. Then the supernatant was fed into a syringe equipped with a filter $(13 \mathrm{~mm} \times 0.22 \mu \mathrm{m})$, and the filtrate $(10 \mu \mathrm{l})$ was injected into the UHPLC-FLD system.

\section{Method validation}

The standard working solution was diluted into a series of concentrations [limit of quantitation (LOQ), 5.0, 10.0, 50.0, 100.0, 150.0 and $200.0 \mu \mathrm{g} / \mathrm{kg}$ ] and added to blank matrix extract. The calibration curve, which was generated by plotting the spiked concentration of piperazine in different blank matrices as the independent variable $(\mathrm{X})$ and the peak area of the piperazine derivative as the dependent variable (Y), was fitted to the equation $Y=a \times$ $X+b$ by a weighted least-squares linear regression.

A series of piperazine standard solutions were added to $2 \mathrm{~g}$ of homogenised blank sample, resulting in final piperazine concentrations of LOQ, 0.5, 1.0 and 2.0 MRL. Multiple spiked samples were analysed by UHPLC-FLD, and the peak areas of the analytes were fitted with equations of the form $Y=a \times X+b$ to calculate the detected piperazine concentrations. The recovery percentage was calculated as the ratio of the detected concentration to the actual added concentration.

Precision was divided into intraday precision and interday precision, and both parameters were evaluated based on relative standard deviations (RSDs). Four spiked concentrations (LOQ, 0.5, 1.0 and 2.0 MRL) were determined using the same calibration curve at different time points in one day, and each spiked concentration was evaluated based on six parallel replicates on the same 
day to determine the intraday RSD. The interday RSD was determined by analysing four spiked concentrations (with six parallel replicates per concentration) on 7 consecutive days using the Horwitz equation, RSD $($ maximum allowable RSD $)=\mathrm{RSD} \times 2 / 3$, where $\mathrm{RSD}=$ $2^{\left(1-0.5^{\circ} \log c\right)}$ and $\mathrm{c}$ is the analyte concentration $(\mathrm{g} / \mathrm{g})$. A method is considered accurate and precise when the recovery is between 80 and $110 \%$ and the RSDs do not exceed RSD ${ }_{\text {max }}$ (European Union, 2002).

The signal-to-noise ratio $(\mathrm{S} / \mathrm{N})$ calculation requires six replicate injections. The limit of detection (LOD) is defined as the lowest analyte concentration that can be detected using the given detector with an $\mathrm{S} / \mathrm{N}$ ratio of $\geq 3$, and the LOQ is the concentration of the analyte when $\mathrm{S} / \mathrm{N} \geq 10$.

Repeatability is one of the indicators used to evaluate chromatographic methods, as it reflects the consistency of the results of multiple injections of the analyte. Six aliquots of the piperazine standard working solution $(1.0 \mu \mathrm{g} / \mathrm{ml})$ were derivatised and assessed using the UHPLC-FLD system, and the RSD values of the retention time and peak area were calculated.

The method developed in this experiment was verified by the measurement and calculation of the linearity, recovery, precision (intraday RSD and interday RSD), LOD and LOQ as stipulated by the European Union (EU) commission decision (European Union, 2002) and the FDA bioanalytical method validation guidelines (U.S. Food and Drug Administration, 2001). The method was also well validated by the stability results and the evaluation of matrix effects.

\section{Results and discussion}

\section{Optimisation of the derivatisation conditions}

We faced a difficult problem in the course of the experiment: piperazine is not easily detected due to its high polarity and its lack of fluorescent group. Piperazine, an amine derivative, can be substituted with common derivatisation reagents such as benzoyl chloride, o-phthalaldehyde and DNS-Cl. Benzoyl chloride is toxic and easily degraded in water (Wada et al., 2015). The product formed using o-phthalaldehyde as the derivatisation reagent has poor stability (Allenmark et al., 1985). When using DNS-Cl as the derivatisation reagent, the efficacy of the derivatisation was better, the conditions were simpler and the obtained derivative had good stability and reproducibility (Dong et al., 2016; Park et al., 2016; Xie et al., 2016). Furthermore, the peak shape of the derivative bearing a dansyl functional group was significantly better than those of the derivatives obtained with the other two derivatisation reagents. Piperazine and DNS-Cl may undergo a single-substitution reaction or a double-substitution reaction, and the derivatives were analysed by mass spectrometry. Piperazine was monosubstituted, generating 1-dansyl piperazine, which is consistent with the results reported by Lin et al. (2010). The reaction equation is shown in Figure 1. We also examined the stability of the derivative at room temperature over a $24 \mathrm{~h}$ period and concluded that the derivative was relatively stable within $12 \mathrm{~h}$ but began to degrade slowly after $16 \mathrm{~h}$.

The derivatisation conditions, including the triethylamine content, the DNS-Cl content and the reaction temperature and time, were optimised, and the control variable method was adopted for analysis. As shown in Figure $2 \mathrm{~A}$, when the triethylamine content is greater than or equal to $0.04 \%$, the peak area of the derivative does not change significantly. When the amount of triethylamine is $0.12 \%$, the peak area of the derivative is relatively large. That is, the optimum amount of triethylamine is $0.12 \%$. Figure $2 \mathrm{~B}$ shows that the peak area of the derivative increases with increasing DNS-Cl content within a certain range. The peak area of the derivative is the largest when $600 \mu \mathrm{l}$ of DNS-Cl is added. The peak area of the derivative was reached at a temperature of $50^{\circ} \mathrm{C}$, and the reaction was closest to being complete at this temperature (see Figure 2C for details). Figure 2D shows that the peak area of the derivative was largest at 20 and $40 \mathrm{~min}$; therefore, the derivatisation time was set at $20 \mathrm{~min}$ to save time. In summary, the optimised derivatisation conditions were $0.12 \%$ triethylamine, $600 \mu \mathrm{l}$ of DNS- $\mathrm{Cl}, 50^{\circ} \mathrm{C}$ and $20 \mathrm{~min}$.

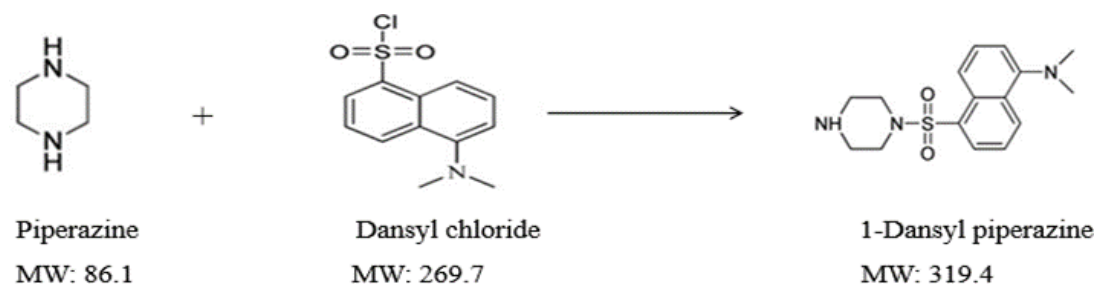

Figure 1. The derivatisation of piperazine with dansyl chloride. 
a

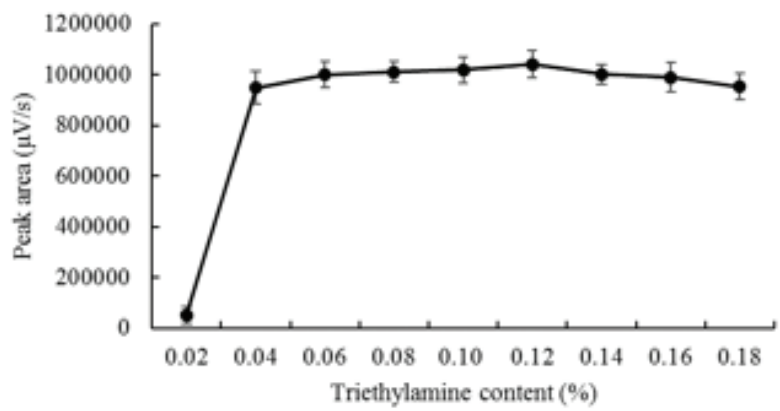

b
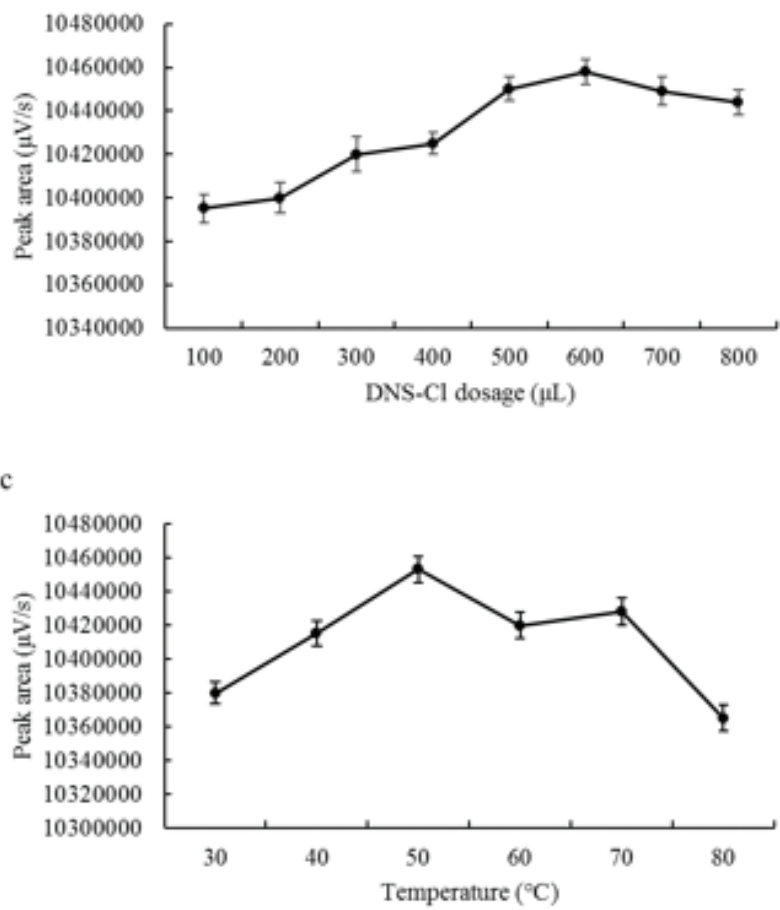

d

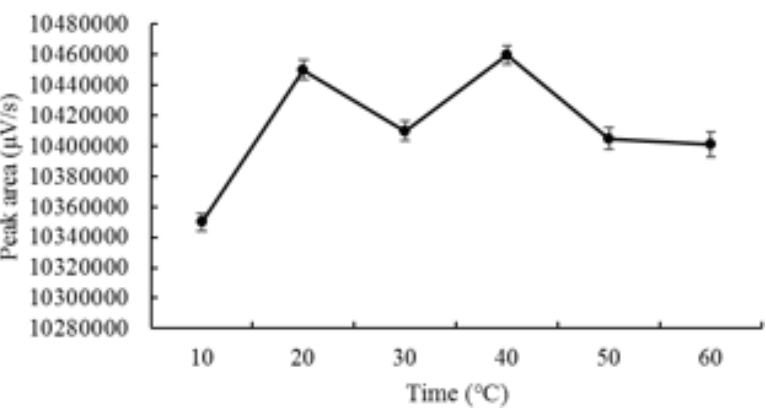

Figure 2. Effects of the triethylamine content (A), the equivalents of dansyl chloride dosage (B), the temperature (C) and the reaction time (D) on the derivatisation reaction.

\section{Optimisation of the ASE conditions}

The pre-treatment of complex samples is often the most challenging part of modern analytical methods.
Many extraction methods, such as microwave-assisted extraction (Staack and Maurer, 2003), ultrasoundassisted extraction (Anna et al., 2017), supercritical fluid extraction (Moawed et al., 2015) and ASE, have been developed, refined and applied. Among these methods, ASE, which combines elevated temperature and pressure with liquid solvents (Richter et al., 1996), is widely used because it has the ability to reduce the amount of extraction solvent required, shorten the sample preparation time and improve the extraction efficiency. ASE also has the advantage of a higher degree of automation compared with other methods (Anna et al., 2017; Moawed et al., 2015; Staack and Maurer, 2003). A target can be subjected to ASE as many times as needed with different solvents or different solvent ratios in the extraction, and all of these parameters can be conveniently and reliably programmed by the user. This automation avoids the interference of artefacts introduced in the manual operation process. Many studies have used ASE to extract target analytes; for example, ASE was used for the rapid extraction of cocaine and benzoylecgonine from coca leaves (Brachet et al., 2015); the extraction of anthraquinones from slimming tea (Wang et al., 2016); and the extraction of phenolic compounds, mainly flavonoids, from species of Passiflora (Gomes et al., 2017).

The recovery rate of the substance to be measured is affected by the extraction solvent and pre-treatment method. Here, $2 \%$ formic acid in water, $2 \%$ formic acid in acetonitrile, $2 \%$ formic acid in water-acetonitrile $(1: 1, \mathrm{~V} / \mathrm{V}), 2 \%$ formic acid in water-acetonitrile $(3: 1, \mathrm{~V} / \mathrm{V})$ and $2 \%$ formic acid in water-acetonitrile $(1: 3, \mathrm{~V} / \mathrm{V})$ were compared in the extraction of piperazine from the biological matrix. The extraction efficiencies achieved using accelerated solvent extraction-solid-phase extraction (ASE-SPE) and liquidliquid extraction-solid-phase extraction (LLE-SPE) techniques were also compared. The data in Table 1 show that the extraction efficiencies achieved by ASE-SPE of the chicken tissues were 35.53-88.82\% (RSD, 1.06-6.42\%) and those using LLE-SPE were $37.39-77.73 \%$ (RSD, 0.68-7.35\%). The extraction efficiencies with the five solvents using ASE-SPE of pork were 45.49-89.99\% (RSD, 1.61-3.51\%) and those using LLE-SPE were 34.79-82.28\% (RSD, 1.88-2.29\%). When the chicken tissues and pork were subjected to ASE-SPE using $2 \%$ formic acid in acetonitrile, the extraction efficiencies were at least $84.96 \%$ (RSD, 1.06\%). Therefore, we extracted piperazine using ASE-SPE with $2 \%$ formic acid in acetonitrile.

ASE is an automated method of extraction using organic solvents at high temperature and high pressure. Increasing the temperature can increase the capacity of the solvent to dissolve the analyte. Increasing the pressure raises the boiling point of the solvent, which is desirable because the solute is much more soluble in the liquid solvent than in the gas. Extraction temperatures from 40 to $120{ }^{\circ} \mathrm{C}$ were analysed, and the peak area of the extract was the highest 


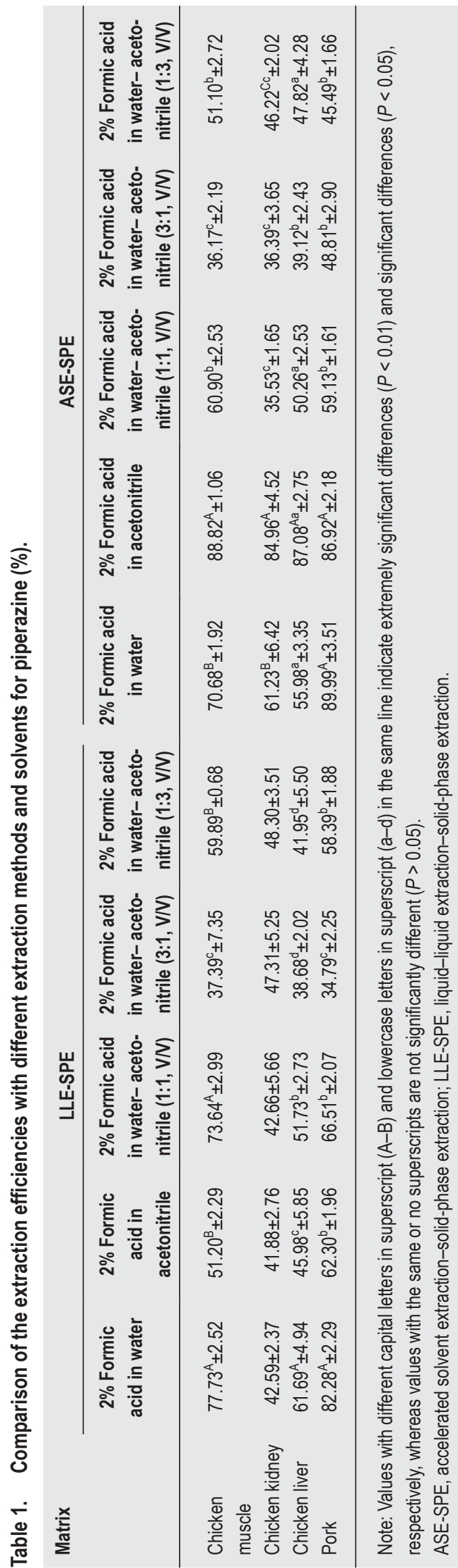

at $80{ }^{\circ} \mathrm{C}$. The extraction pressure is typically set to 1,500 psi, and minor variations around this value have little effect on the extraction efficiency. In light of earlier studies, we chose $80^{\circ} \mathrm{C}$ and $1,500 \mathrm{psi}$ as the final extraction conditions for the ASE.

\section{Selection of the SPE columns and optimisation of the SPE conditions}

Sample purification is conducted to remove impurities and improve the sensitivity and accuracy of the analysis results. SPE is considered a good choice for purification and enrichment of trace components, especially in samples containing complex impurities. SPE does not require a large number of mutually incompatible solvents, and no emulsification occurs during the treatment. This technique uses a highly efficient and highly selective adsorbent (stationary phase) that significantly reduces solvent usage and simplifies the pre-treatment process. In this study, three types of cation-exchange columns (PCX, MCX and Strata-X-C) were compared. The recovery percentages with the PCX column ( $3 \mathrm{ml} / 60 \mathrm{mg}$, Agela Technologies Corp., CA, USA) and the MCX column ( $3 \mathrm{ml} / 60 \mathrm{mg}$, Waters Corp., Milford, MA, USA) were less than $70 \%$. The PCX column is a weak cation-exchange column, and it showed insufficient enrichment of piperazine (substantial baseline noise was observed), and the purification effect of the MCX column on weak alkaline compounds, such as piperazine, is inadequate (interference from an impurity peak is observed). The Strata-X-C column had an obvious purification effect, and the recoveries were greater than $80 \%$. This result may be related to the type of filler, the packing technique and the pore size of the column. Based on evaluation of searchable publications (Lee et al., 1997; Lin et al., 2010; Llasera and García-Cicourel, 2017; McClintic et al., 2003; Park et al., 2016; Sakaguchi et al., 2016) and preliminary experiments, methanol and $2 \%$ formic acid in water were used as the activation and balance solutions, methanol and 0.1 $\mathrm{mol} / \mathrm{L}$ hydrochloric acid in water were used as leachates and $10 \%$ ammonia methanol was used as the eluent. We found that $2 \mathrm{ml}$ of $0.1 \mathrm{~mol} / \mathrm{L}$ hydrochloric acid in water and methanol could eliminate the impurities adsorbed by the SPE column without rinsing out the analytes. A high concentration of piperazine (2.0 MRL) in the sample could be eluted with $9 \mathrm{ml}$ of $10 \%$ ammonia in water and methanol.

\section{Optimisation of the chromatographic conditions}

The key to achieve good separation in chromatographic analysis is the selection and optimisation of the chromatographic conditions. By tuning these conditions, chromatographic peaks with high symmetry and good resolution can be achieved, which is essential for the analysis of trace analytes. This study compared the separation efficacies of an Acquity UPLC BEH amide column $(2.1 \mathrm{~mm} \times 100 \mathrm{~mm}, 1.7 \mu \mathrm{m})$, 
an Acquity UPLC BEH C18 column $(2.1 \mathrm{~mm} \times 100 \mathrm{~mm}$, $1.7 \mu \mathrm{m})$ and an Acquity UPLC HSS T3 column $(2.1 \mathrm{~mm} \times$ $100 \mathrm{~mm}, 1.8 \mu \mathrm{m})$. The retention time of the derivatives on the $\mathrm{C} 18$ column and the amide column was reasonable; however, the chromatographic peaks showed substantial tailing, which may be caused by insufficient inertness of the surface of the column filler or interactions between the silanol group and the amines. The HSS T3 column is filled with tightly bound silica gel particles, which effectively retain polar substances and do not strongly retain nonpolar substances; thus, a good chromatographic peak shape can be obtained for piperazine, a highly polar alkaline compound. There was no interference from impurity peaks around the peak of the analyte, and no leading peaks or tailing was observed. We examined the separation efficacies of three different ratios of the ultrapure water-acetonitrile mobile phase system (10:90, $15: 85$ and 20:80, V/V). When the composition of the mobile phase was 15:85, the retention time of the derivative was reasonable, and the target peak was not affected by interferants.

Isocratic and gradient elution differ in whether the composition ratio and the flow rate of the mobile phase change. We used a reversed-phase chromatographic column specifically designed for the separation and retention of polar substances. For highly polar piperazine, using a high-proportion organic phase has a strong elution ability and can provide a good separation effect. In this study, isocratic elution was performed with solutions A (ultrapure water) and B (acetonitrile) at $0.2 \mathrm{ml} / \mathrm{min}$, and the ratio was $15: 85(\mathrm{~V}: \mathrm{V})$. The target peak was well shaped and well separated from the baseline, and the retention time was $3.207 \mathrm{~min}$, as shown in Figure 3. Figure 4 shows that the chromatographic peak of the derivative is well separated from any interfering impurities, and the target peak is symmetrical. When the piperazine standard was added to blank matrix at a concentration of $50.0 \mu \mathrm{g} / \mathrm{kg}$, the retention times of the derivatives from chicken muscle, kidney and liver were 3.195, 3.223 and $3.224 \mathrm{~min}$, respectively, and that from pork was $3.194 \mathrm{~min}$.

\section{Method validation}

Any new test method needs to be verified according to certain requirements to ensure its scientific rigour before its application. The method validation was performed in accordance
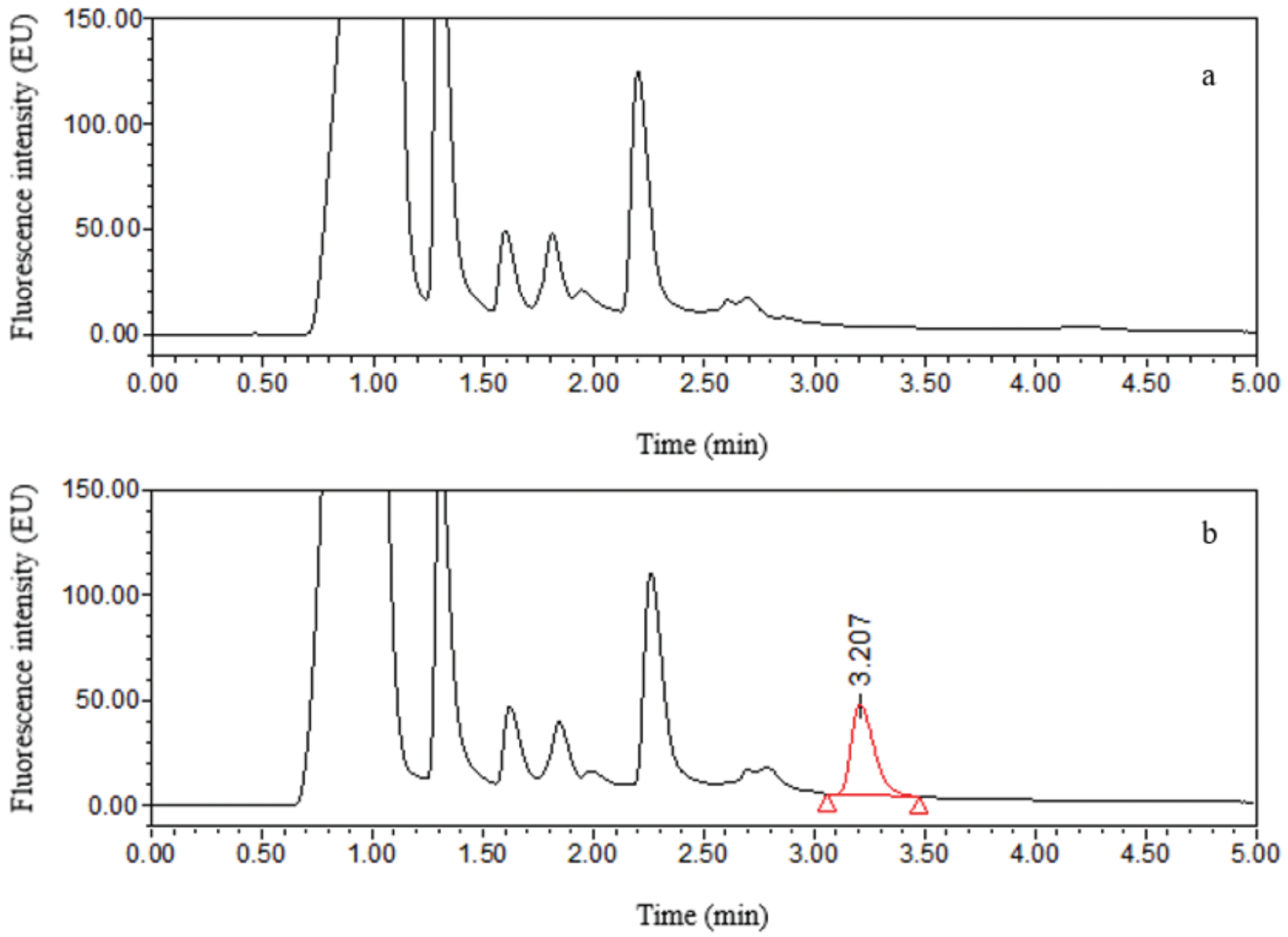

Figure 3. Ultrahigh-performance liquid chromatograms of dansyl chloride standard (A) and $50 \mu g / k g$ piperazine standard (B). 

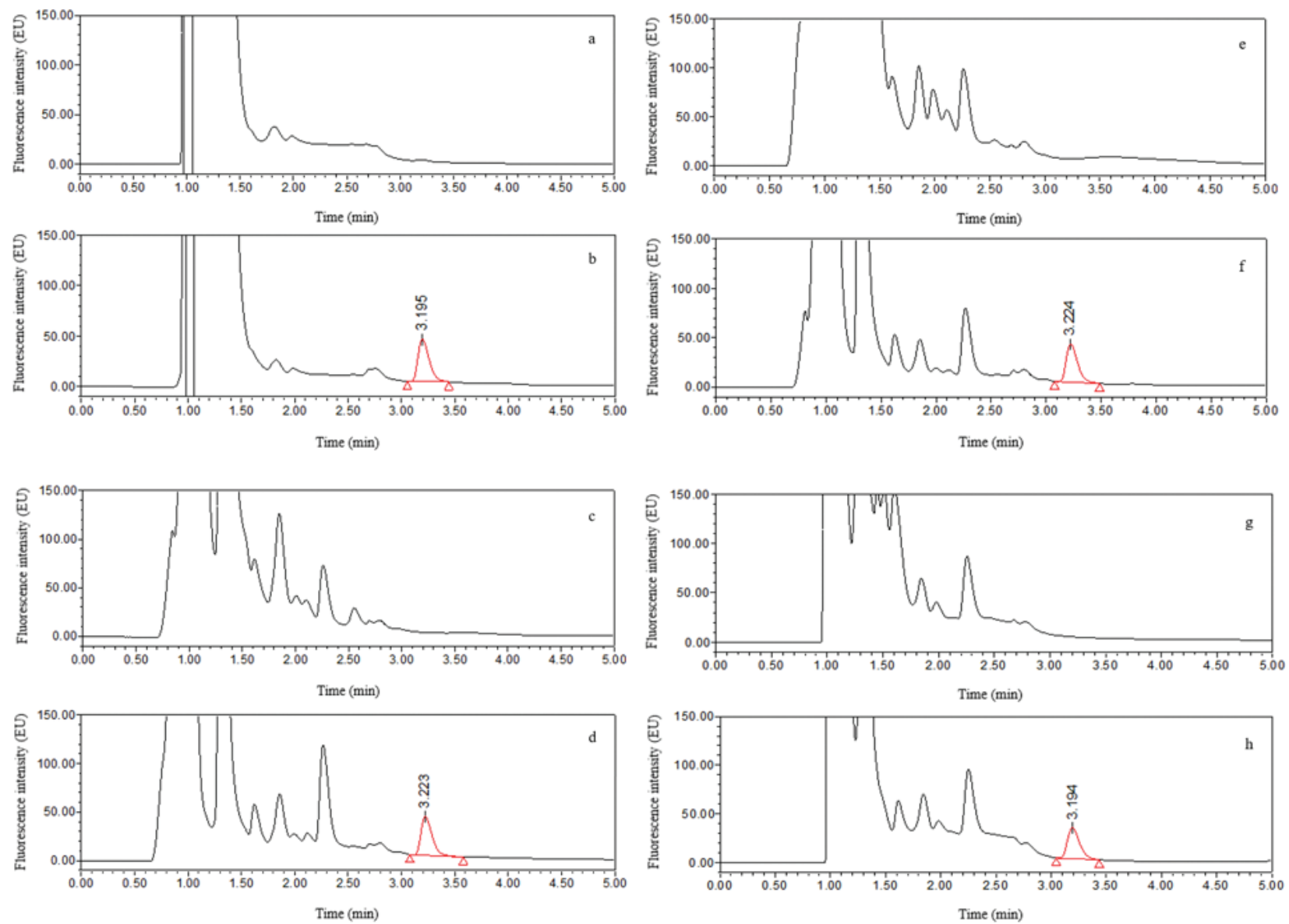

Figure 4. Ultrahigh-performance liquid chromatograms of blank matrix and blank matrix spiked with $50 \mu \mathrm{g} / \mathrm{kg}$ piperazine standard. (A) Blank chicken muscle; (B) blank chicken muscle spiked with 50 $\mu$ g/kg piperazine standard; (C) blank chicken kidney; (D) blank chicken kidney spiked with $50 \mu \mathrm{g} / \mathrm{kg}$ piperazine standard; (E) blank chicken liver; (F) blank chicken liver spiked with 50 $\mu \mathrm{g} / \mathrm{kg}$ piperazine standard; (G) blank pork; and (H) blank pork spiked with $\mathbf{5 0} \mu \mathrm{g} / \mathrm{kg}$ piperazine standard.

Table 2. The linear range, linear regression equation and coefficient of determination of chicken tissues and pork.

\begin{tabular}{lccc} 
Matrix & Linearity range $(\boldsymbol{\mu g} / \mathbf{k g})$ & Linear regression equation & Coefficient of determination $\left(\mathbf{R}^{2}\right)$ \\
\hline Chicken muscle & $1.80-200.0$ & $y=54,151 \times-6,597$ & 0.9997 \\
Chicken kidney & $3.50-200.0$ & $y=52,702 \times-19,427$ & 0.9991 \\
Chicken liver & $2.40-200.0$ & $y=54,264 v-33,486$ & 0.9995 \\
Pork & $1.85-200.0$ & $y=52,581 \times-4,523.4$ & 0.9994
\end{tabular}

with the EU Commission decision (European Union, 2002) and the FDA guidelines for bioanalytical method validation (U.S. Food and Drug Administration, 2001).

The concentrations of piperazine in blank chicken tissues (muscle, kidney and liver) and pork were in the range of LOQ-200.0 $\mu \mathrm{g} / \mathrm{kg}$, and the peak area of the piperazine derivative showed a good linear relationship with the concentration of added piperazine. The linear range, linear regression equation and $R^{2}$ are listed in Table 2 .
Recovery is an important indicator of the accuracy of an analytical method, and it reflects the matrix effects in the method. The same homogeneous sample is repeatedly measured, and the degree of closeness between the measured values is the precision. Precision represents the repeatability of an analysis and is expressed by the RSD. The spiked concentrations of piperazine in the blank sample were equal to the LOQ, 0.5, 1.0 and 2.0 MRL, and the calculated recovery rate and precision are shown in Table 3. 
Table 3. Recovery and precision of piperazine added to blank chicken tissues and pork $(n=6)$.

\begin{tabular}{|c|c|c|c|c|c|}
\hline Matrix & Level added $(\mu \mathrm{g} / \mathrm{kg})$ & Recovery (\%) & RSD (\%) & Intraday RSD (\%) & Interday RSD (\%) \\
\hline \multirow[t]{4}{*}{ Chicken muscle } & 1.80 & $88.02 \pm 1.62$ & 1.84 & 4.03 & 5.84 \\
\hline & 50.0 & $88.31 \pm 3.27$ & 3.70 & 2.87 & 4.25 \\
\hline & $100.0^{a}$ & $96.75 \pm 3.13$ & 3.24 & 3.28 & 4.47 \\
\hline & 200.0 & $99.77 \pm 3.46$ & 3.47 & 4.24 & 4.02 \\
\hline \multirow[t]{4}{*}{ Chicken liver } & 3.50 & $79.64 \pm 7.46$ & 5.63 & 6.71 & 7.77 \\
\hline & 50.0 & $85.83 \pm 2.21$ & 2.57 & 3.90 & 4.74 \\
\hline & $100.0^{a}$ & $86.31 \pm 2.92$ & 3.38 & 2.82 & 5.69 \\
\hline & 200.0 & $83.71 \pm 1.17$ & 1.40 & 3.36 & 3.96 \\
\hline \multirow[t]{4}{*}{ Chicken kidney } & 2.40 & $80.10 \pm 1.62$ & 2.02 & 6.35 & 6.59 \\
\hline & 50.0 & $89.96 \pm 2.76$ & 3.07 & 4.18 & 5.99 \\
\hline & $100.0^{a}$ & $86.64 \pm 1.05$ & 1.21 & 3.80 & 7.07 \\
\hline & 200.0 & $89.86 \pm 1.02$ & 1.14 & 2.62 & 3.52 \\
\hline \multirow[t]{4}{*}{ Pork } & 1.85 & $87.10 \pm 2.23$ & 2.56 & 4.68 & 5.06 \\
\hline & 50.0 & $92.70 \pm 1.87$ & 2.01 & 2.50 & 2.58 \\
\hline & $100.0^{a}$ & $94.71 \pm 2.98$ & 3.15 & 6.25 & 6.44 \\
\hline & 200.0 & $90.07 \pm 2.23$ & 2.48 & 4.68 & 5.08 \\
\hline
\end{tabular}

The lowest concentration required to detect the presence of the analyte being measured is the LOD, and the lowest concentration required to quantify the analyte being measured is the LOQ. Both parameters can be obtained by calculating $\mathrm{S} / \mathrm{N}$ values and are important indicators of the sensitivity of the method. The LODs of piperazine in chicken muscle, kidney and liver were $0.50,1.20$ and $0.80 \mu \mathrm{g} / \mathrm{kg}$, respectively, and the LOQs were $1.80,3.50$ and $2.40 \mu \mathrm{g} / \mathrm{kg}$, respectively. The LOD of piperazine in pork was $0.62 \mu \mathrm{g} / \mathrm{kg}$, and the LOQ was $1.85 \mu \mathrm{g} / \mathrm{kg}$.

The repeatability reflects the consistency of multiple analysis results of the analyte. Six independent measurements were made under different experimental conditions. The RSDs of the retention time and peak area of the piperazine derivative were 0.10 and $0.18 \%$, respectively.

The stability of the standard is a key factor in ensuring that the test results are accurate. We studied the stability of the piperazine standard solution under different temperature conditions. The standard is stable during storage for 2 months in the dark at $-70{ }^{\circ} \mathrm{C}$ or for 1 month at $4{ }^{\circ} \mathrm{C}$. At room temperature $\left(25^{\circ} \mathrm{C}\right)$, the compound degraded gradually after $15 \mathrm{~h}$. If long-term storage is required, we recommend protecting the compound from light and storing it at $-70{ }^{\circ} \mathrm{C}$.

\section{Evaluation of matrix effects}

Matrix effects are defined as the effects of other components in the matrix on the accuracy of the determination of the mass or concentration of the analyte. Such effects
Table 4. Recovery (\%) of piperazine at different concentrations from different matrices.

\begin{tabular}{lcccc}
$\begin{array}{l}\text { Level } \\
\text { added } \\
(\boldsymbol{\mu} \mathrm{g} / \mathrm{kg})\end{array}$ & $\begin{array}{c}\text { Chicken } \\
\text { muscle }\end{array}$ & $\begin{array}{c}\text { Chicken } \\
\text { kidney }\end{array}$ & $\begin{array}{c}\text { Chicken } \\
\text { liver }\end{array}$ & Pork \\
\cline { 2 - 5 } & 102.40 & 92.70 & 95.23 & 94.59 \\
\hline $.5 \mathrm{MRL}$ & 105.27 & 91.28 & 92.32 & 96.84 \\
$1.0 \mathrm{MRL}$ & 107.50 & 89.32 & 92.66 & 97.23 \\
$2.0 \mathrm{MRL}$ & & & &
\end{tabular}

are inevitable and can be divided into enhancement and suppression effects. This study investigated the matrix effects at three concentrations (0.5, 1.0, and 2.0 MRL), and the matrix effects of each sample are shown in Table 4. The recoveries from the various samples ranged from 83 to $110 \%$, which was consistent with the research of Matuszewski and Chavezeng (2003). Chicken muscle showed an enhancement effect, and the other matrices showed suppression effects. This study optimised the method to decrease the influence of the matrix effect by adding piperazine into blank matrix.

\section{Comparison of different detection methods}

In previous studies, our research team developed UHPLC-ESI/ME/MS (Xie et al., 2016) and GC-ESI/MS/ MS (Wang et al., 2017) methods for the determination of piperazine residues. Compared to complex systems that need to be combined with expensive mass spectrometry, it is more economical and broadly applicable to use an independent UHPLC system for detection. 
The application of UHPLC in this experiment provides suitable chromatographic separation for piperazine detection. Piperazine can be detected using a FLD at trace levels only after being derivatised with a group giving it high optical density or the capacity for induced light emission. Previous reports have described various derivatisation reagents for piperazine. For instance, in the research reported by Navaneeswari and Reddy (2012), 4-chloro-7-nitrobenzofuran was used as a derivatisation reagent to determine the levels of piperazine in pharmaceutical preparations. Acetic anhydride was exploited as the derivatisation reagent in Wang et al.'s (2017) study on the determination of piperazine residues in chicken and pig tissues. In the studies by Lin et al. (2010) and Park et al. (2016) on determining piperazine contents, DNS-Cl was selected as the derivatisation reagent. We evaluated and optimised the derivatisation conditions (equivalents of the reagent, reaction time and reaction temperature) to obtain a stable, monosubstituted product, and the signals met the requirements after the targets were captured by the detector. Compared with the results of Park et al.'s (2016) study, the retention time was shorter (17.4 min versus $3.2 \mathrm{~min}$ ), and our method achieved lower LODs and LOQs due to the optimisation of the pre-treatment methods and chromatographic conditions. In particular, the implementation of ASE in the pre-treatment has many advantages, such as reducing the time required and simplifying the operation. Comparison of the recoveries obtained with ASE-SPE and LLE-SPE, we found that ASE demonstrated better extraction efficiency (see Table 1 for details). In addition to the innovation of the method, this platform has obvious advantages over other methods in terms of reagent usage and ease of operation.

\section{Application of the method}

To evaluate the applicability and reliability of the newly developed method, the researchers purchased 20 chicken samples (the muscle, kidney and liver of each chicken were sampled and tested) and 15 pork samples from a local supermarket for analysis. The detected concentrations in chicken muscle, kidney and liver and pork were $13.5-21.6,19.2-29.8,17.9-23.6$ and $12.6-21.5 \mu \mathrm{g} / \mathrm{kg}$, respectively; none of the samples contained piperazine above the MRL set by the FDA $(100.0 \mu \mathrm{g} / \mathrm{kg})$. Thus, the present method can be applied as a sophisticated method to quantify piperazine in chicken and pig tissue samples.

\section{Conclusions}

In this study, the highly selective and sensitive determination of piperazine residues in chicken tissues (muscle, kidney and liver) and pork was achieved using a newly developed ASE-SPE-UHPLC-FLD method coupled with precolumn derivatisation (DNS-Cl). The experimental parameters of this newly established approach are sound and meet the requirements of the confirmatory criteria set by the EU. In addition, the practicability and reliability of this method have been demonstrated in the analysis of samples from local supermarkets.

\section{Acknowledgements}

This research was financially supported by the China Agriculture Research System (CARS-41-G23), the National Natural Science Foundation of China (31302009), the Priority Academic Program Development of Jiangsu Higher Education Institutions (PAPD), Jiangsu Agriculture Science and Technology Innovation Fund (CX (18) 3022) and the Yangzhou University High-End Talent Support Program.

\section{Conflict of interest}

The authors declare that there are no conflicts of interest with any financial organisation regarding the material discussed in this article.

\section{Funding}

This research was financially supported by the China Agriculture Research System (CARS-41-G23), the National Natural Science Foundation of China (31302009), the Priority Academic Program Development of Jiangsu Higher Education Institutions (PAPD), Jiangsu Agriculture Science and Technology Innovation Fund (CX (18) 3022) and the Yangzhou University High-End Talent Support Program.

\section{Compliance with ethical standards}

All experimental procedures were conducted in strict compliance with the recommendations of the Guide for the Care and Use of Laboratory Animals of Jiangsu Province and of the Animal Care and Use Committee of the Ministry of agriculture and rural areas of China. All efforts were made to minimize animal suffering.

\section{References}

Allenmark, S., Bergström, S. and Enerback, L., 1985. A selective postcolumn o-phthalaldehyde-derivatization system for the determination of histamine in biological material by high-performance liquid chromatography. Analytical Biochemistry 144(1): 98-103. https://doi.org/10.1016/0003-2697(85)90089-2

Anna, P.G.N., Tiwari, S., Tube, J., Vyas, V. and Qureshi, G., 2017. Ultrasound assisted-synthesis and biological evaluation of piperazinylprop-1-en-2-yloxy-2H-chromen-2-ones as cytotoxic agents. Drug Design and Discovery 14: 1195-1205. https://doi. org/10.2174/1570180814666170322154750 
Brachet, A., Rudaz, S., Mateus, L., Christen, P. and Veuthsy, J.L., 2015. Optimisation of accelerated solvent extraction of cocaine and benzoylecgonine from coca leaves. Journal of Separation Science 24(10/11): 865-873. https://doi. org/10.1002/1615-9314(20011101)24:10/11

Brennan, K.A., Lake, B., Hely, L.S., Jones, K., Gittings, D., Colussi-mass, J., Fitzmaurice, P.S., Lea, R.A. and Schenk, S., 2007. N-benzylpiperazine has characteristics of a drug of abuse. Behavioural Pharmacology 18(8): 785-790. https://doi. org/10.1097/fbp.0b013e3282f18d8f

Ciaccio, L.L., Missan, S.R., Mcmullen, W.H. and Grenfell, T.C., 1957. Nonaqueous titration of 1,4-Disubstituted piperazines. Analytical Chemistry 29(11): 1670-1673. https://doi.org/10.1021/ ac60131a032

Demirci, S., Hayal, T.B., kiratli, B., Sisli, H.B., Demirci, S., Sahin F. and Dogan, A., 2019. Design and synthesis of phenylpiperazine derivatives as potent anticancer agents for prostate cancer. Chemical Biology \& Drug Design 94: 1-12. https://doi. org/10.1111/cbdd.13575

Dong, S., Yan, Z. and Yang, H. ..., 2016. A sensitive precolumn derivatization method for determination of piperazine in vortioxetine hydrobromide using a C8 column and high-performance liquid chromatography-mass spectrometry. Analytical Sciences 32(12): 1333-1338. https://doi.org/10.2116/analsci.32.1333

European Union, 2002. Commission decision of 2002/657/EC implementing council directive 96/23/EC concerning the performance of analytical methods and the interpretation of results, Official Journal of the European Union, London. Available at: https:/ec.europa.eu/ health/files/eudralex/vol-5/reg_2010_37/reg_2010_37_en.pdf.

Glamkowski, E.J., StrupczewskiJ., Wolf, E. and Woodward, D.L., 1974. Antihypertensive activity of 1-dimethylphosphinylmethyl4-arylpiperazines. Journal of Medicinal Chemistry 17: 1008-1009. https://doi.org/10.1021/jm00255a021

Gomes, S.V.F., Portugal, L.A., Anjos, J.P., Jesus, O.N., Oliveira, E.J., David, J.P. and David, J.M., 2017. Accelerated solvent extraction of phenolic compounds exploiting a box-behnken design and quantification of five flavonoids by HPLC-DAD in passiflora species. Microchemical Journal 132: 28-35. https://doi.org/10.1016/j. microc.2016.12.021

Hayat, F., Zia-ur, R. and Muhammad Haleem, K., 2017. Two new heteroleptic ruthenium (II) dithiocarbamates: synthesis, characterization, DFT calculation and DNA binding. Journal of Coordination Chemistry 70(2): 279-295. https://doi.org/10.1080/ 00958972.2016.1255328

Lalka, D. and Bardos, T.J., 2010. Reactions of 2,2-dimethylaziridine-type alkylating agents in biological systems I: colorimetric estimation and stability in physiological media. Journal of Pharmaceutical Sciences 62(8): 1294-1298. https://doi.org/10.1002/jps.2600620813

Lee, S., Casteel, D.A. and Fleckenstein, L., 1997. Specific gas chromatographic analysis of diethylcarbamazine in human plasma using solid-phase extraction. Journal of Chromatography B 704(1-2): 181-185. https://doi.org/10.1016/s0378-4347(97)00424-6

Lin, H., Tian, Y., Zhang, Z., Wu, L. and Chen, Y., 2010. Quantification of piperazine phosphate in human plasma by high-performance liquid chromatography-electrospray ionization tandem mass spectrometry employing precolumn derivatization with dansyl chloride. Analytica Chimica Acta 664(1): 40-48. https://doi. org/10.1016/j.aca.2010.02.003

Llasera, M.P. and García-Cicourel, A.R., 2017. On-line SPE chromatography with spectrophotometric diode array detection as a simple and advantageous choice for the selective trace analysis of benzo(a) anthracene degradation products from microalgae. Talanta 165: 584-592. https://doi.org/10.1016/j.talanta.2017.01.011

Marchetti, M.L., Errecalde, J. and Mestorino, N., 2012. Effect of 1-(1-naphthylmethyl)-piperazine on antimicrobial agent susceptibility in multidrug-resistant isogenic and veterinary Escherichia coli field strains. Journal of Medical Microbiology 61: 786-792. https://doi.org/10.1099/jmm.0.040204-0

Matuszewski, B.K. and Chavezeng, C.M., 2003. Strategies for the assessment of matrix effect in quantitative bioanalytical methods based on HPLC-MS/MS. Analytical Chemistry 75(13): 3019-3030. https://doi.org/10.1021/ac020361s

McClintic, C., Remick, D.M., Perterson, J.A. and Risley, D.S., 2003. Novel method for the determination of piperazine in pharmaceutical drug substances using hydrophilic interaction chromatography and evaporative light scattering detection. Journal of Liquid Chromatography \& Related Technologies 26(18): 3093-3104. https://doi.org/10.1081/JLC-120025426

Ministry of Agriculture of the People's Republic of China. 2002. Maximum residue limit of veterinary drugs in animal food Bulletin No. 235 (Appendix 4), Beijing. Available at: https://www. moa.gov.cn/.

Moawed, E.A., Abulkibash, A.B. and El-shahat, M.F., 2015. Synthesis of tannic acid azo polyurethane sorbent and its application for extraction and determination of atrazine and prometryn pesticides in foods and water samples. Environmental Nanotechnology, Monitoring \& Management 3: 61-66. https://doi.org/10.1016/j. enmm.2015.02.001

Navaneeswari, R. and Reddy, P.R., 2012. Analytical method for piperazine in an active pharmaceutical ingredient using chemical derivatization and HPLC-UV. Journal of Chemical and Pharmaceutical Research 4(6): 2854-2859. https://doi. org/10.1080/10826071003608959

Nnaji, N.J.N., Ujam, O.T., Ibisi, N.E., Ani, J.U., Onuegbu, T.O. and Olasunkanmi, L.O., 2017. Morpholine and piperazine based carboxamide derivatives as corrosion inhibitors of mild steel in $\mathrm{HCl}$ medium. Journal of Molecular Liquids 230: 652-661. https://doi. org/10.1016/j.molliq.2017.01.075

Park, J.A., Zhang, D., Kim, S., Cho, S., Jeong, D., Kim, J., Shim, J., Abd el-aty, A.M. and Shin, H., 2016. Development of a high-performance liquid chromatography with fluorescence detection method for quantification of piperazine in animal products by using precolumn derivatization. Food Chemistry 196: 1331-1337. https://doi.org/10.1016/j.foodchem.2015.10.081

Peek, H.W. and Landman, W.J.M., 2011. Coccidiosis in poultry: anticoccidial products, vaccines and other prevention strategies. Veterinary Quarterly 31(3): 143-161. https://doi.org/10.1080/016 52176.2011.605247

Richter, B.E., Jones, B.A., Ezzell, J.L., Porter, N.L., Avdalovic, N. and Pohl, C.,1996. Accelerated solvent extraction: a technique 
for sample preparation. Analytical Chemistry 68(6): 1033-1039. https://doi.org/10.1021/ac9508199

Sakaguchi, Y., Kinumi, T. and Takatsu, A., 2016. Quantification of peptides using N囚terminal isotope coding and C-terminal derivatization for sensitive analysis by micro liquid chromatography-tandem mass spectrometry. Journal of Mass Spectrometry 51: 1111-1119. https://doi.org/10.1002/jms.3845

Silva, D.P.B., Florentino, I.F., Oliveira, L.P., Lino, R.C., Galdino, P.M., Menegatti, R. and Costa, E.A., 2015. Anti-nociceptive and anti-inflammatory activities of 4-[(1-phenyl-1H-pyrazol-4-yl) methyl] 1-piperazine carboxylic acid ethyl ester: a new piperazine derivative. Pharmacology Biochemistry and Behavior 137: 86-92. https://doi.org/10.1016/j.pbb.2015.08.008

Staack, R.F. and Maurer, H.H., 2003. Toxicological detection of the new designer drug 1-(4-methoxyphenyl) piperazine and its metabolites in urine and differentiation from an intake of structurally related medicaments using gas chromatography-mass spectrometry. Journal of Chromatography B 798(2): 333-342. https://doi.org/10.1016/j.jchromb.2003.10.004

Standardization Administration of the People's Republic of China, 2008. Water for analytical laboratory use-specification and test methods, Standards Press of China, Beijing. Available at: https:// www.sac.gov.cn.

The European Medicines Agency, 2010. Commission regulation (EU) No. 37/2010 of 22 December 2009 on pharmacologically active substances and their classification regarding maximum residue limits in foodstuffs of animal origin, Official Journal of the European Union, London. Available at: https://ec.europa.eu/ health/files/eudralex/vol-5/reg_2010_37/reg_2010_37_en.pdf.

The Japan Food Chemical Research Foundation, 2015. Maximum residue limits (MRLs) list of agricultural chemicals in foods, National Printing Bureau, Tokyo. Available at: https://www. m5.ws001.squarestart.ne.jp/foundation/agrdtl.php?a_inq=53500.
U.S. Food and Drug Administration, 2001. Guidance for industry: bioanalytical method validation, Government Printing Office, Washington, DC. Available at: https://www.fda.gov/downloads/ Drugs/GuidanceComplianceRegulatoryInformation/Guidances/ UCM070107.pdf.

U.S. Food and Drug Administration, 2014. CFR-Code of federal regulations title 21 part 556 tolerances for residue of new animal drugs in food, Government Printing Office, Rockville, MD. Available at: https:/www.accessdata.fda.gov/scripts/cdrh/cfdocs/cfCFR/ CFRSearch.cfm?CFRPart287=556\&showFR=1.

Wada, M., Yamahara, K., Ikeda, R., Kikura-Hanajiri, R., Kuroda, N. and Nakashima, K., 2015. Simultaneous determination of N-benzylpiperazine and 1-(3-trifluoromethylphenyl) piperazine in rat plasma by HPLC-fluorescence detection and its application to monitoring of these drugs. biomedical chromatography 26 : 21-25. https://doi.org/10.1002/bmc.1619

Wang, B., Pang, M., Xie, X., Xie, K., Zhang, Y., Cui, L., Zhao, X., Wang, Y., Shi, H., Guo, Y., Wang, R., Zhang, G., Dai, G. and Wang, J., 2017. Quantification of piperazine in chicken and pig tissues by gas chromatography-electron ionization tandem mass spectrometry employing pre-column derivatization with acetic anhydride. Journal of Chromatography A 1519: 9-18. https://doi. org/10.1016/j.chroma.2017.08.079

Wang, N., Su, M., Liang, S. and Sun, H., 2016. Investigation of six bioactive anthraquinones in slimming tea by accelerated solvent extraction and high performance capillary electrophoresis with diode-array detection. Food Chemistry 199: 1-7. https://doi. org/10.1016/j.foodchem.2015.11.083

Xie, K., Liu, Y., Sun, L., Pang, M., Xie, X., Gao, Q., Wang, B., Zhang, Y., Wang, R., Zhang, G., Dai, G. and Wang, J., 2016. Quantification of piperazine in chicken muscle by ultra-Performance liquid chromatography-electrospray ionization tandem mass spectrometry. Food Analytical Methods 1-9.https://doi.org/10.1007/s12161-016-0717-x 